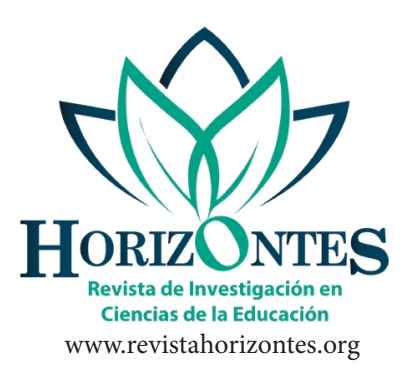

\title{
Estilos de Liderazgo y Clima Organizacional en tiempos de emergencia sanitaria
}

\author{
Leadership Styles and Organizational Climate in times of health emergency
}

Estilos de liderança e clima organizacional em tempos de emergência de saúde

\section{Lucia Lourdes De La Cruz-Urrutia}

1ldel@ucvvirtual.edu.pe

https://orcid.org/0000-0002-4318-700X

Universidad César Vallejo, Lima-Perú

Artículo recibido 25 de octubre 2021, arbitrado y aceptado 29 de noviembre 2021 y publicado 28 de enero 2022

RESUMEN

ABSTRACT

RESUMO

La presente investigación analiza el liderazgo y clima organizacional en tiempos de emergencia sanitaria, tomando como muestra poblacional a los directivos de un Instituto de Educación superior. Para ello, se estableció un diseño no experimental, transversal y correlacional, cuyos instrumentos fueron: el cuestionario sobre Liderazgo de los Directivos y el cuestionario sobre Clima Organizacional. Cabe señalar que la confiabilidad se estableció en base a una prueba piloto aplicada a 30 estudiantes, obteniendo un Alfa de Cronbach superior a 0.74 en todas sus dimensiones; por ello, se procedió a tomar la encuesta a una muestra de 100 estudiantes, para la que se aplicó un muestreo no probabilístico censal. Finalmente, el análisis estadístico arroja una relación positiva $(\mathrm{r}=0,62 ; \mathrm{p}=0.000$ $\mathrm{r}=0,52 ; \mathrm{p}=0.000 \mathrm{r}=0,43 ; \mathrm{p}=0.000$ ) con un nivel de correlación moderada entre las variables de estudio, es decir, se concluye que hay una relación positiva y estadísticamente significativa.

Palabras clave: Liderazgo; Gestión; Pandemia; Correlación; Ambiente de trabajo
This research analyzes the leadership and organizational climate in times of health emergency, taking as a population sample the directors of an Institute of Higher Education. For this, a non-experimental, cross-sectional and correlational design was established, the instruments of which were: the questionnaire on Leadership of Directors and the questionnaire on Organizational Climate. It should be noted that the reliability was established based on a pilot test applied to 30 students, obtaining a Cronbach's Alpha higher than 0.74 in all its dimensions; Therefore, the survey was taken from a sample of 100 students, for which a non-probabilistic census sampling was applied. Finally, the statistical analysis shows a positive relationship $(r=0.62$; $\mathrm{p}=0.000 \mathrm{r}=0.52 ; \mathrm{p}=0.000 \mathrm{r}=0.43$; $\mathrm{p}=0.000)$ with a moderate level of correlation between the study variables, that is, it is concluded that there is a positive and statistically significant relationship.

Key words: Leadership; Management; Pandemics; Correlation; Work environment
Esta pesquisa analisa a liderança e o clima organizacional em momentos de emergência de saúde, tomando como amostra populacional os diretores de uma Instituição de Ensino Superior. Para tanto, foi estabelecido um desenho não experimental, transversal e correlacional, cujos instrumentos foram: o questionário de Liderança de Diretores e o questionário de Clima Organizacional. Ressalta-se que a confiabilidade foi estabelecida a partir de um teste piloto aplicado a 30 alunos, obtendo-se um Alfa de Cronbach superior a 0,74 em todas as suas dimensões; portanto, a pesquisa foi realizada a partir de uma amostra de 100 alunos, para a qual foi aplicada uma amostra censitária não probabilística. Por fim, a análise estatística mostra uma relação positiva $(\mathrm{r}=0,62 ; \mathrm{p}=0,000 \mathrm{r}=$ $0,52 ; \mathrm{p}=0,000 \mathrm{r}=0,43 ; \mathrm{p}=0,000)$ com um nível moderado de correlação entre as variáveis de estudo, ou seja, concluise que existe uma relação positiva e estatisticamente significativa.

Palavras chave: Liderança; Gestão; Pandemia; Correlação; Ambiente de trabalho 


\section{INTRODUCCIÓN}

La denominada nueva normalidad, evidenció diversas falencias en los sistemas de salud públicos, también la ineficacia de los gobiernos en la distribución de riquezas y el poco énfasis sobre sectores indispensables para la vida humana. Así, la realidad educativa fue una de las más afectadas, en la medida que los gobiernos recién estaban implementando recursos tecnológicos materiales, por lo que surgían dicotomías como: qué hacer para llegar a la mayor parte de la población, cómo reducir las barreras del distanciamiento y generar espacios que pudieran ser eficaces para el desarrollo del proceso de enseñanza y aprendizaje (Barbosa et al., 2021).

En ese sentido, los gobiernos intentaron; en primera instancia, generar planes de emergencia que consideren las limitaciones materiales, por ello se enfocaron en dotar a la población escolar de dispositivos electrónicos como computadoras y celulares, sin dejar de mencionar artefactos de difusión masiva como la televisión (Kochen, 2020); en segunda instancia, reformar los programas, planes y metas establecidos anualmente; por último, dar apertura a una reflexión sobre los contenidos y herramientas que configurarían los ahora denominados Entornos Virtuales de Aprendizaje (EVA).

Pero la realidad de los acontecimientos no fue tan armoniosa, en la medida que en pleno 2021 muchos gobiernos todavía no logran suplir los requerimientos materiales para lleva a cabo una eficiente educación a distancia, mucho menos existe entre las instituciones educativas un panorama claro de hacia dónde apunta la brújula en temas de planificación. En este punto, deberíamos de reflexionar sobre el motivo o causa que impide que todas las instituciones estén alineadas a las disposiciones gubernamentales. Así, uno de los problemas no tan evidenciable es la desconexión existente entre la entidad gubernamental educativa y las diversas jurisdicciones que la componen (Macancela y Paredes, 2021).

En efecto, el problema no es únicamente gubernamental, sino de gestión, lo cual nos remite a determinados directivos a nivel micro y macro que se vieron obligados a poner en práctica todas las estrategias de liderazgo para que sus instituciones se actualicen en las nuevas normativas y el empleo de las herramientas digitales. La coyuntura mostró la importancia del liderazgo asumido por los directores educativos, en la medida que fue la condición que permitió el cumplimiento de la entrega de recursos y que sus instituciones no se queden atrás en la aplicación de las nuevas disposiciones.

En ese sentido, el liderazgo de los directores educativos fue y es fundamental para canalizar y desplegar las disposiciones gubernamentales en pandemia, pero todavía hay mucho por hacer, ya que el deber fundamental de todo líder es generar climas organizacionales que permitan el trabajo en equipo y el desarrollo de cada uno de sus elementos (Blanco et al., 2021).

Así, el nuevo desafío es regresar a un tema indispensable en la presencialidad: tratar de relacionar el estilo de liderazgo asumido por los líderes educativos y el clima organizacional. Justo ahora que los docentes presentan mayores desafíos, como el integrar en sus prácticas herramientas y estrategias digitales, sin dejar de mencionar el agotamiento físico y mental que ello implica (Bada et al., 2020). 
Por todo lo mencionado, la relación entre el liderazgo y clima organizacional en instituciones educativas se convierten en un tema de investigación interesante, puesto que, muchas investigaciones sugieren que el ambiente organizacional tiene una fuerte influencia en las percepciones y el comportamiento de los empleados (Pereira y Solís, 2019). A medida que aumenta la capacidad y habilidad de un buen liderazgo se optimiza el clima organizacional dentro de una institución. En efecto, estudios por resonancia magnética funcional (FMRI) demuestran que las emociones negativas dentro del entorno laboral pueden incrementar los niveles de cortisol y desarrollar síntomas psicosomáticos de estrés, irritabilidad, ansiedad, depresión, entre otros.

Por otro lado, es indispensable definir nuestras variables, por ello concebimos; en primer lugar, al clima organizacional como una serie de características que influyen en el ambiente de trabajo y el comportamiento de los trabajadores, sus relaciones interpersonales yla percepción individual del trabajador sobre su contexto organizacional (Berberoglu, 2018); en segundo lugar, al liderazgo no solo como una definición simbólica de dirigir, sino de motivar comportamientos en otras personas, más aún si los efectos motivacionales de una buena dirección tienen efectos en el cambio conductual y la toma de decisiones de los estudiantes (Delgado, 2019).

En esa dirección, se debe ser cuidadoso al referirse al término liderazgo; debido a que existen diferentes tipos como: el conductual o vertical, situacional, transaccional o transformacional (Reyes y Rojas, 2017). La importancia de esta distinción radica en que no todos los liderazgos logran un buen clima organizacional, a pesar de la funcionalidad que puedan lograr en las instituciones. Aunque la adopción de un estilo de liderazgo es algo complejo, en la medida que las instituciones son parte de una dirección mayor que limita sus elecciones.

Recogiendo todo lo expuesto, es propósito de este estudio analizar la relación existente entre los estilos de liderazgo y el clima organizacional, tomando como base un estudio descriptivo no experimental que considera a los directivos de una Institución de Educación Superior en tiempos de emergencia sanitaria. Así mismo, se debe señalar que la hipótesis de estudio se enmarcó en la existencia de una relación estadísticamente significativa entre el liderazgo de los directivos y el clima organizacional.

\section{MÉTODO}

La investigación fue de tipo descriptiva, de corte transversal-correlacional. Se caracteriza por tener un doble propósito: la descripción y el análisis (Rodríguez y Mendivelso, 2018). Gracias a este diseño es posible manipular las variables, investigar sin ninguna transformación de la realidad o situación nueva.

La población de estudio fue de 140 estudiantes, de entre 19 y 55 años de edad de un Instituto de Educación Superior, a través de un muestreo de tipo no probabilístico por conveniencia fue seleccionada una muestra de 76 estudiantes del sexo femenino y 24 estudiantes del sexo masculino; para la selección de la muestra se tuvo en cuenta tres criterios de inclusión: haberse matriculado en período regular, asistencia continua a clases y un desarrollo adecuado del instrumento de aplicación al momento de la recolección de datos. 
Para la medición de la variable de estudio, estilos de liderazgos de los directivos, se seleccionó el cuestionario sobre estilos de liderazgo de directivos y para la variable clima organizacional el cuestionario sobre clima organizacional, ambos cuestionarios se encuentran validados a nivel internacional y son confiables para su aplicación. Sin embargo, previo a su aplicación se realizó un proceso de validación completa de ambos instrumentos.

En la primera etapa se aplicó una prueba piloto a 30 estudiantes de la institución educativa, en ambos instrumentos mencionados, posterior a ello, se utilizó la prueba Alfa de Cronbach; los resultados para el cuestionario sobre liderazgo de los directivos mostraron un alfa de $\alpha=0,74$ para la dimensión autoritario, $\alpha=0,84$ dimensión democrático $y$ $\alpha=0,82$ para la dimensión liberal, y esto indicó una confiabilidad moderada.

Por otro lado, el cuestionario sobre clima organizacional mostró un alfa de $\alpha=0,94$ demostrando una alta confiabilidad. En conclusión, ambos instrumentos son consistentes y confiables para su aplicabilidad en el contexto de estudio.

Una vez validados los instrumentos, se procedió a la recolección de datos correspondientes, para la que se procesaron y tabularon los datos utilizando el software estadístico Statistical Package for Social Sciences (SPSS) a fin de interpretar los resultados con la mayor objetividad posible. También se tabularon los instrumentos en dos bases de datos diferentes, realizaron análisis de frecuencia, tanto relativa como absoluta, se codificaron los niveles totales. Para determinar la normalidad en la distribución de los datos, por el tamaño de la muestra $(\mathrm{n}=100)$, se utilizó la prueba estadística de Kolmogorov-Smirnov; para la prueba de hipótesis, se usó el estadístico Rho de Spearman, a fin de extraer un coeficiente de asociación entre los estilos de liderazgo de los directivos y clima organizacional.

Por otro lado, se analizaron las variables de estudio y sus respectivas dimensiones. Así mismo, con el objetivo de determinar la normalidad en la distribución de los datos, por el tamaño de la muestra $(n=100)$, se utilizó la prueba estadística de Kolmogórov-Smirnov. Además, dado que los datos no se distribuyeron de forma normal, se usó el estadístico Rho de Spearman, a fin de extraer un coeficiente de relación entre las dos variables de estudio.

\section{RESULTADOS Y DISCUSIÓN}

A continuación, se presentan los principales resultados a nivel descriptivo para cada una de las variables de estudio, estilos de liderazgo de los directivos y clima organizacional.

En cuanto, estilo de liderazgo autoritario y clima organizacional en la Tabla 1 se observa que un grupo representativo del $28 \%$ de los estudiantes evaluados percibe un liderazgo autoritario de nivel alto y a la vez la existencia de un clima organizacional de nivel alto. En tanto que observa que solo el $16 \%$ considera que el ejercicio de un liderazgo autoritario de nivel bajo reflejará también un clima organizacional bajo. 
Tabla 1. Contingencia estilo de liderazgo autoritario y clima organizacional.

\begin{tabular}{llcccc}
\hline \multirow{2}{*}{ Niveles } & & \multicolumn{3}{c}{ Clima organizacional } & \multirow{2}{*}{ Total } \\
\hline Estilo de liderazgo autoritario & Bajo & Bajo & Promedio & Alto & \\
& Promedio & 5 & 6 & 0 & 25 \\
& Alto & 3 & 19 & 19 & 40 \\
Total & & $\mathbf{2 7}$ & $\mathbf{2 6}$ & $\mathbf{4 7}$ & $\mathbf{1 0 0}$ \\
\hline
\end{tabular}

En cuanto al estilo de liderazgo democrático y clima organizacional en la Tabla 2, se observa que un grupo representativo del $33 \%$ de los estudiantes evaluados percibe un liderazgo democrático promedio y a la vez la existencia de un clima organizacional de nivel alto. En tanto, solo el $3 \%$ considera percibir un liderazgo democrático de nivel alto que se verá reflejado en un clima organizacional de nivel bajo.

Tabla 2. Contingencia estilo de liderazgo democrático y clima organizacional.

\begin{tabular}{|c|c|c|c|c|c|}
\hline \multirow{2}{*}{ Niveles } & & \multicolumn{3}{|c|}{ Clima organizacional } & \multirow{2}{*}{ Total } \\
\hline & & Bajo & Promedio & Alto & \\
\hline \multirow[t]{3}{*}{ Estilo de liderazgo autoritario } & Bajo & 16 & 5 & 4 & 25 \\
\hline & Promedio & 8 & 18 & 33 & 59 \\
\hline & Alto & 3 & 3 & 10 & 16 \\
\hline Total & & 27 & 26 & 47 & 100 \\
\hline
\end{tabular}

En cuanto al estilo de liderazgo liberal y clima organizacional en la Tabla 3 , se observa que un grupo representativo de $25 \%$ de los estudiantes evaluados percibe un liderazgo liberal de nivel promedio y a la vez la existencia de un clima organizacional de nivel alto. En tanto solo el 3\% considera percibir un liderazgo liberal de nivel alto que se verá reflejado en un clima organizacional bajo.

Tabla 3. Contingencia estilo de liderazgo liberal y clima organizacional.

\begin{tabular}{llcccc}
\hline \multicolumn{1}{c}{ Niveles } & & \multicolumn{2}{c}{ Clima organizacional } & & Total \\
& & Bajo & Promedio & Alto & \\
\hline Estilo de liderazgo autoritario & Bajo & 19 & 16 & 13 & 48 \\
& Promedio & 5 & 4 & 25 & 34 \\
& Alto & 3 & 6 & $\mathbf{4 7}$ & 18 \\
\hline Total & & $\mathbf{2 7}$ & $\mathbf{2 6}$ & $\mathbf{1 0 0}$ \\
\hline
\end{tabular}


Sobre los estilos de liderazgo de los directivos se pudo observar que existe un grupo representativo del $35 \%$ de los estudiantes evaluados que percibe como predominante el estilo de liderazgo autoritario, en tanto que solo el $18 \%$ de estos alumnos encuestados manifiesta como predominante el estilo de liderazgo liberal, y finalmente, apenas el 16\% de los sujetos del estudio mencionan como predominante el estilo de liderazgo democrático. Por lo tanto, se puede concluir que el estilo de liderazgo de los directivos que predomina en la institución educativa es el autoritario.

Sobre el clima organizacional se pudo observar que si bien existe un grupo representativo del $47 \%$ de los estudiantes evaluados que percibe que existe un alto nivel en el clima organizacional; no es menos importante el hecho de que el $27 \%$ de los alumnos encuestados manifiesta que existe un bajo nivel en el clima organizacional y finalmente el $26 \%$ de los sujetos del estudio menciona que este clima se encuentra en un nivel promedio. Estos datos señalan que el clima organizacional de la institución educativa aún debe mejorar.

Sobre la relación de las variables de estudio: Se observa que existe un grupo representativo del $28 \%$ de estudiantes evaluados que percibe el clima organizacional en un nivel alto y a la vez como predominante el estilo de liderazgo autoritario.
Así mismo, solo el $10 \%$ de los alumnos encuestados considera que el clima organizacional se ubica en un nivel alto y a la vez como predominante el estilo democrático y finalmente apenas el $9 \%$ de los sujetos del estudio considera que el clima organizacional se encuentra en un nivel alto y a la vez percibe como predominante el estilo de liderazgo liberal.

A partir de estos datos, se puede inferir que, de acuerdo a la percepción de los sujetos participantes de la investigación, de los tres estilos analizados, es el estilo autoritario el que se encuentra más asociado a un mejor funcionamiento del clima organizacional en la institución educativa.

\section{Prueba de hipótesis}

H0: No existe relación significativa entre el liderazgo de los directivos y el clima organizacional en el Instituto de Educación Superior de la Región Callao, 2019.

Ha: Existe relación significativa entre el liderazgo de los directivos y el clima organizacional en el Instituto de Educación Superior de la Región Callao, 2019.

Tabla 4. Nivel de significancia y regla de decisión de la hipótesis.

Nivel de significancia

$\alpha=0.05=5 \%$ de margen máximo de error

\section{Regla de decisión}

$p \geq \alpha \rightarrow$ se rechaza la hipótesis nula $\mathrm{H}_{0}$

No existe relación significativa entre el liderazgo de los directivos y el clima organizacional

$\mathrm{p}<\mathrm{\alpha} \rightarrow$ se acepta la hipótesis nula $\mathrm{Ha}$

Existe relación significativa entre el liderazgo de los directivos y el clima organizacional 
Tabla 5. Coeficiente de correlación de Spearman de las variables liderazgo de los directivos y clima organizacional.

\begin{tabular}{|c|c|c|c|}
\hline \multicolumn{3}{|c|}{ Liderazgo de los directivos } & \multirow{2}{*}{$\begin{array}{c}\text { Clima organizaciona } \\
, 628^{* *}\end{array}$} \\
\hline Rho de Spearman & Autoritario & Correlación & \\
\hline & & Sig. (bilateral) & 000 \\
\hline & Democrático & Correlación &, $628^{\star *}$ \\
\hline & & Sig. (bilateral) & ,000 \\
\hline & Liberal & Correlación &, $628^{\star *}$ \\
\hline & & Sig. (bilateral) & ,000 \\
\hline
\end{tabular}

**. La correlación es significativa en el nivel 0,01 (2 colas).

Decisión estadística. Se encontró un nivel de significación (valor $\mathrm{p})$ menor a $0,05(\mathrm{p}=<0,05)$, lo que permite señalar que la relación es estadísticamente significativa; por lo tanto, se rechaza la hipótesis nula y se acepta la hipótesis alternativa o hipótesis del investigador. Es decir, existe relación positiva y significativa entre el liderazgo de los directivos y el clima organizacional en el Instituto de Educación Superior de la Región Callao, 2019 (Tabla 4).

Descripción del grado de relación entre las variables. Los resultados del análisis estadístico dan cuenta en todos los casos de la existencia de relación positiva y estadísticamente significativa $(\mathrm{r}$ $=0,62 ; \mathrm{r}=0,52 ; \mathrm{r}=0,43 ; \mathrm{p}=<0,05 \mathrm{r})$ con un nivel de correlación moderada entre las variables liderazgo de los directivos y clima organizacional (Tabla 5).

Esto infiere que mientras se optimice el liderazgo dentro de la organización también mejorará el clima organizacional.
Descripción del grado de relación entre las variables. Los resultados del análisis estadístico dan cuenta en todos los casos de la existencia de una relación positiva, con un nivel de correlación moderada $(r=0,65$ y $r=0,59 \mathrm{p}=<0,05)$ entre los estilos de liderazgo autoritario y democrático y con un nivel de correlación bajo $(r=0,36)$ entre el estilo de liderazgo liberal y la dimensión recompensa del clima organizacional.

Decisión estadística. Se encontró un nivel de significación (valor p) menor a 0.05 , lo que permite señalar que la relación es significativa, por lo tanto, se rechaza la hipótesis nula y se acepta la hipótesis alternativa. Es decir, existe relación positiva y significativa entre el liderazgo de los directivos con la dimensión recompensa del clima organizacional en un Instituto de Educación Superior de la Región Callao. 
Tabla 6. Correlación entre el liderazgo de los directivos y la dimensión recompensa del clima organizacional.

\begin{tabular}{lllc}
\hline Estilos de liderazgo de los directivos & & Recompensa \\
\hline \multirow{2}{*}{ Rho de Spearman } & Autoritario & Coeficiente de correlación & $0,653^{* *}$ \\
& & Sig. (bilateral) &, 000 \\
& \multirow{2}{*}{ Democrático } & Coeficiente de correlación &, $595^{* *}$ \\
& & Sig. (bilateral) &, 000 \\
& Liberal & Coeficiente de correlación &, $369^{* *}$ \\
& & Sig. (bilateral) &, 000 \\
\hline
\end{tabular}

**. La correlación es significativa en el nivel 0,01 (2 colas).

Tabla 7. Correlación entre el liderazgo de los directivos y la dimensión relaciones interpersonales del clima organizacional.

\begin{tabular}{|c|c|c|c|}
\hline \multicolumn{3}{|c|}{ Estilos de liderazgo de los directivos } & \multirow{2}{*}{$\begin{array}{c}\text { Relaciones interpersonales } \\
, 455^{\star *}\end{array}$} \\
\hline Rho de Spearman & Autoritario & Coeficiente de correlación & \\
\hline & & Sig. (bilateral) & 000 \\
\hline & Democrático & Coeficiente de correlación & $430^{* *}$ \\
\hline & & Sig. (bilateral) & 000 \\
\hline & Liberal & Coeficiente de correlación & $256^{\star}$ \\
\hline & & Sig. (bilateral) & 010 \\
\hline
\end{tabular}

**. La correlación es significativa en el nivel 0,01 (2 colas).

*. La correlación es significativa en el nivel 0,05 ( 2 colas).

Descripción del grado de relación entre las variables. Los resultados del análisis estadístico dan cuenta en todos los casos de la existencia de una relación positiva, con un nivel de correlación moderada $(r=0,45$ y $r=0,43)$ entre los estilos liderazgo autoritario y democrático y con un nivel de correlación bajo $(r=0,25)$ entre el estilo de liderazgo liberal y la dimensión relaciones interpersonales del clima organizacional. Decisión estadística: Se encontró un nivel de significación (valor p) menor a 0,05 lo que permite señalar que la relación es significativa, por lo tanto, se rechaza la hipótesis nula y se acepta la hipótesis alternativa. Es decir, existe relación positiva y significativa entre el liderazgo de los directivos con la dimensión relaciones interpersonales del clima organizacional (Tabla 7). 
Tabla 8. Correlación entre el liderazgo de los directivos y la dimensión autonomía del clima organizacional.

\begin{tabular}{lllc}
\hline Estilos de liderazgo de los directivos & & Relaciones interpersonales \\
\hline \multirow{2}{*}{ Rho de Spearman } & Autoritario & Coeficiente de correlación &, $513^{* *}$ \\
& & Sig. (bilateral) &, 000 \\
& \multirow{2}{*}{ Democrático } & Coeficiente de correlación &, $430^{* *}$ \\
& Sig. (bilateral) &, 000 \\
& Liberal & Coeficiente de correlación &, $675^{* *}$ \\
& Sig. (bilateral) &, 000 \\
\hline
\end{tabular}

\section{Descripción del grado de relación entre las} variables. Los resultados del análisis estadístico dan cuenta en todos los casos de la existencia de una relación positiva y con un nivel de correlación moderada $(r=0,51 ; r=0,43$ y $r=0,67)$ entre los estilos de liderazgo de los directivos autoritario, democrático y liberal con la dimensión autonomía del clima organizacional.

Decisión estadística. Se encontró un nivel de significación (valor p) menor a 0,05 , lo que permite señalar que la relación es significativa, por tanto, se rechaza la hipótesis nula y se acepta la hipótesis alternativa. Es decir, existe relación positiva y significativa entre el liderazgo de los directivos con la dimensión autonomía del clima organizacional en el Instituto de Educación Superior de la Región Callao, 2019.

Así, los resultados de la presente investigación muestran que predomina el estilo de liderazgo autoritario con un $35 \%$ y el estilo liberal con un $18 \%$; por lo tanto, el estilo de liderazgo predominante entre los directivos en la institución educativa es el autoritario. El estilo de liderazgo del director se hace relevante por influenciar en el comportamiento de otras personas con el fin de alcanzar los objetivos institucionales e individuales.

\section{Discusión}

Los resultados abren una reflexión: ¿el liderazgo autoritario es positivo o negativo dentro de las organizaciones? Un liderazgo autoritario se caracteriza por un líder que ordena sin consultar, que presiona en el cumplimiento de las obligaciones y responsabilidades a sus subordinados. Para Miras y Longás (2020), los cánones jerárquicos que han caracterizado la administración educativa son obsoletos y ameritan la búsqueda de cambios que respondan a las demandas sociales. Domingo (2019) señala que este liderazgo puede llevar a resultados contraproducentes, por lo que hoy en día se ha abandonado la visión del liderazgo individual, en aras de lograr el desarrollo de "liderazgos transformativos".

De hecho, así exista un modelo de liderazgo óptimo dentro de la institución, si no se tiene un buen clima organizacional, es probable que esta institución fracase en sus logros académicos. Así, Rivera y Cegarra (2016) encontraron que en el contexto educativo donde no se propicia el clima organizacional se da una suerte de desmotivación grupal en los integrantes del grupo, es decir, tanto docentes, directivos y estudiantes no encuentran motivos internos para cumplir sus objetivos; se tiene lo contrario cuando los directivos y docentes 
propician el buen clima organizacional, un ambiente de armonía que impulsa el sentido de pertenencia hacia la institución educativa donde se trabaja. (Rivera y Cegarra, 2016).

En cuanto al liderazgo democrático, Villalba (2017) refiere que este tipo de liderazgo dentro de una organización es fundamental para fomentar la colaboración en equipo, la comunicación, los acuerdos, la responsabilidad y cumplimiento de obligaciones por parte de estudiantes y docentes, sin dejar de mencionar los es pacios de toma de decisiones en la que todos pueden participar por igual. Según Schultze et al. (2019), el tipo de liderazgo señalado pordría evitar situaciones de exclusión participativa que generen un clima de hostilidad posterior.

Por otro lado, los estilos de liderazgo, democrático, autoritario y liberal tienen una relación positiva y moderada con el clima organizacional, además, esta relación fue estadísticamente significativa, es decir, a medida que aumentto uno $\mathrm{u}$ otro tipo de liderazgo también tiende a aumentar el clima laboral. Wen (2019) notó que mientras más autoritarios y dictatoriales son los líderes, menos era el desempeño de los integrantes de la administración de la escuela así como la motivación.

Así mismo, Pilligua et al., (2019) señala que las instituciones, independientemente del rubro, tienen la necesidad de propiciar un clima de trabajo agradable con un liderazgo colaborativo, ya que ello influye en los resultados colectivos.Sin embargo, no todos los estilos de liderazgo tienen una relación positiva con el clima laboral. Así, algunos auotore han demostrado que el liderazgo transformacional crea un clima organizacional positivo y deseable, tanto que se fomenta el sentido de pertenencia, lo cual evidencia que cuando los líderes mejoran sus actitudes hacia la organización también mejora la actitud de los trabajadores.

Además, la relación existente entre el clima organizacional y el liderazgo no solo afectará las actitudes y emociones que sientan tanto docentes como directores, sino también repercutirán en la forma de sentir, pensar y actuar de los educandos. Así, Caliman et al., (2020) indica que las instituciones educativas tienen el deber de construir entornos que sirvan al desarrollo del liderazgo juvenil, con la finalidad de hacerlos partícipes de iniciativas que puedan aportar a la ciudadanía, ya que las buenas relaciones, la confianza y un ambiente de trabajo positivo generan mayor participación.

Cavalcante (2018) añade que todo clima organizacioal debe comprender condiciones materiales y sociambientales que estimulen la participación de sus miembros de la comunidad educativa.El último se presenta como un mediador entre el liderazgo y otras variables laborales importantes como: la satisfacción, la productividad, el estilo de trabajo y el rendimeinto. (Moslehpour, et al., 2019).

Se encontró una fuerte correlación estadística entre liderazgo y clima organización con respecto a la productividad de los trabajadores. En otro estudio realizado a una muestra de 3180 encuestados de 52 empresas sudafricanas los autores reflejan la importancia de incluir al clima organizacional en estudios multivariados tal como lo precisan Sethibe y Steyn, (2018) es decir, no se podría enriquecer una investigación sobre productividad laboral sin incluir entre sus variables al clima organizacional, más efectivo que las recopenzas contingentes como: el salario, bonificaciones, obsequios, entre 
otros. (Sethibe y Steyn, 2018). Adicionalmente, Nguyen et al., (2021) manifiesta que para medir la productividad también es necesario encontrar los factores de crisis y subsanarlos.

Así mismo, investigaciones han mostrado una fuerte correlación entre las variables del presente estudio, es el caso de Gregory y Willis (2019) a la hora de afirmar que el liderazgo es un fenómeno interactivo que lleva a la búsqueda de objetivos comunes;sin embargo, la diferencia con nuestro estudio radica en que para ellos el liderazgo democrático es relevante para el clima organizacional, pero para nosotros lo es más el liderazgo desinteresado y comunitario, aquel que se desarrolla cuando el director se preocupa por las necesidades e interereses personales y profesionales de los maestros y genera un clima organizacional positivo e impulsa a que los docentes puedan participar de forma activa.

De acuerdo al análisis y discusión de los resultados se rechaza la hipótesis nula y se acepta la hipótesis de estudio, puesto que, existe correlación positiva y estadísticamente significativa entre los estilos de liderazgo y el clima organizacional.

\section{CONCLUSIONES}

El proceso de enseñanza y aprendizaje se adscribe a una institución en la que se generan relaciones interpersonales que configuran las circunstancias en las que todos sus miembros se desenvolverán. En ese sentido, es indispensable que toda organización educativa considere la relación entre el liderazgo y el clima organizacional, entendiendo que el primero repercutirá en el clima organizacional en el que se desempeñan los actores del proceso pedagógico. Por lo que es muy importante no solo enfocarse en la programación de los planes de estudios y los contenidos que se impartirán, sino en la convivencia a través de un análisis de las necesidades del capital humano y estudiantil.

\section{REFERENCIAS}

Bada Quispe, O.F., Salas Sánchez, R., y Castillo Saavedra, E., y Arroyo Rosales, E., y Carbonell García, C. (2020). Estrés laboral y clima organizacional en docentes peruanos. MediSur, 18(6),1138-1144. https://www.redalyc.org/ journal/1800/180065571009/

Barbosa, M., Garcia, L., y Monte Coelho Frota, A. (2021). Devir-professor brasileiro em tempos de pandemia. Childhood \& Philosophy, 17, 1-18. https://www.redalyc.org/ journal/5120/512066359013/512066359013.pdf

Berberoglu, A. (2018). Impact of organizational climate on organizational commitment and perceived organizational performance: empirical evidence from public hospitals. Health Services Research, 18. https://bmchealthservres. biomedcentral.com/articles/10.1186/s12913018-3149-z

Blanco-López, S., y Cerdas-Montano, V., y GarcíaMartínez, J. (2021). Clima organizacional en centros educativos públicos costarricenses: un análisis multifactorial. Revista Educación, 45(1),1-18. https://www.redalyc.org/ journal/440/44064134020/

Caliman, G., Guimaraes, I., Lucena, J., Ivaldo A., y Gonçalves, V. (2020). Youth leadership and global citizenship: alternatives for peacebuilding in Brazilian public schools. Avaliação e Políticas Públicas em Educação, 28(108),1-23. https:// www.redalyc.org/articulo.oa?id=399563646012

Cavalcante, P., Guedes, S., Loiola, E., Debiasi, A. y da Silva, A. (2018). Aprendizagem individual, suporte organizacional e desempenho percebido: Um estudo com docentes universitários. Educação em Revista, 34.https:// www.redalyc.org/articulo.oa?id=399365005021 
Delgado, D. (2019). Role of higher education in Colombia in peacebuilding. Revista Electrónica en Educación y Pedagogía, 3(5), 147-153. https:// www.redalyc.org/articulo.oa?id=573962080009

Domingo, J. (2019). Una dirección escolar con capacidad de liderazgo pedagógico. Revista Mexicana de Investigación educativa, 24(82), 897-911. HTTPS://WWW.REDALYC.ORG/ ARTICULO.OA?ID $=14062838012$

Gregory, A. y Willis, P. (2019). Communication and leadership go hand in hand: Strategic Leadership and Communication Management [Leadership strategy and communication management], EUNSA, 322.

Kochen, G. (2020). La gestión directiva o el liderazgo educativo en tiempos de pandemia. Innovaciones Educativas, 22(33), 9-12. https:// www.scielo.sa.cr/scielo.php?pid=S2215$41322020000200009 \& \mathrm{script}=\mathrm{sci}_{\text {- }}$ abstract\&tlng=es

Macancela, D. y Paredes, M. (2021). El liderazgo educativo frente a la crisis sanitaria y su repercusión en el sistema organizacional del Colegio Augusto Mendoza Moreira. 593 Digital Publisher CEIT, 6(2), 5-24. https://dialnet. unirioja.es/servlet/articulo? codigo $=7896335$

Miras, J. y Longás, J. (2020). Liderazgo pedagógico y liderazgo ético: perspectivas complementarias de la nueva dirección escolar. REXE. Revista de Estudios y Experiencias en Educación, 19(41), 287-305. https://www.redalyc.org/articulo. oa?id=243165542017

Moslehpour, M., Altantsetseg, P., Mou, W. y Wong, W - K. (2019). Organizational Climate and Work Style: The Missing. Sustainability, 11(125). https://www.mdpi.com/2071-1050/11/1/125

Nguyen, V., Nguyen, Q., Dinh, T. y Nguyen, Le D. (2021). Critical factors affecting construction labor productivity: A comparison between perceptions of project managers and contractors.
Cogent Business \& Management, 8,11-17. https://www.tandfonline.com/doi/full/10.1080/ 23311975.2020.1863303

Pereira, A. y Solís, D. (2019). Factores del clima organizacional(Caso: Instituciones educacionales de San Carlos). Journal of Chemical Information and Modeling, 53(9), 1689-1699. https://www.redalyc.org/ jatsRepo/5636/563662173007/index.html

Pilligua, L., Cristhian, F. y Arteaga, F. (2019). El clima laboral como factor clave en el rendimiento productivo de las empresas. Estudio caso: Hardepex Cía. Cuadernos Latinoamericanos de Administración, 15(28), https://www.redalyc.org/articulo. oa? id=409659500007

Reyes, D. y Rojas, R. (2017). Estilo de liderazgo predominante en organizaciones sociales dedicadas a la educación ambiental. Revista Venezolana de Gerencia, 22(80), 1-9. http:// www.redalyc.org/articulo.oa?id=29055967007

Rivera, C., Cegarra, O., Vergara, H. y Matos, Y. (2016). Clima Organizacional en el Contexto Educativo. Revista Scientific, 1(2), 316-339

Rodríguez, M. y Mendivelso, F. (2018). Diseño de Investigación de Corte Transversal. Sanitas, 21(3), 141-146. https://www.unisanitas.edu.co/ Revista/68/07Rev\%20Medica\%20Sanitas\%20 21-3_MRodriguez_et_al.pdf

Villalba, M. (2017). El liderazgo Democrático: Una Aproximación Conceptual. NNOVA Research Journal, 2(4), 155-162. https://revistas.uide.edu. ec/index.php/innova/article/view/210/273

Wen, H. (2019). Effect of leadership style and organizational climate on employees' food safety and hygiene behaviors in the institutional food service of schools. Food Science and Nutrition. https://onlinelibrary.wiley.com/doi/ full/10.1002/fsn3.1056 